\title{
Statistical complexity measures as telltale of relevant scales in emergent dynamics of spatial systems
}

\author{
A. Arbona ${ }^{1}$, C. Bona ${ }^{1}$, B. Miñano ${ }^{1}$, and A. Plastino ${ }^{2}$ \\ ${ }^{1}$ IAC $^{3}$-UIB, Mateu Orfila, Ctra. de Valldemossa km 7.5, 07122, \\ Palma, Spain \\ ${ }^{2}$ National University La Plata, Physics Institute \\ (IFLP-CCT-CONICET) C.C. 727, 1900 La Plata, Argentina
}

November 5, 2018

\begin{abstract}
The definition of complexity through Statistical Complexity Measures (SCM) has recently seen major improvements. Mostly, effort is concentrated in measures on time series. We propose a SCM definition for spatial dynamical systems. Our definition is in line with the trend to combine entropy with measures of structure (such as disequilibrium). We study the behaviour of our definition against the vectorial noise model of Collective Motion. From a global perspective, we show how our SCM is minimal at both the microscale and macroscale, while it reaches a maximum at the ranges that define the mesoscale in this model. From a local perspective, the SCM is minimum both in highly ordered and chaotic areas, while it reaches a maximum at the edges between such areas. These characteristics suggest this is a good candidate for detecting the mesoscale of arbitrary dynamical systems as well as regions where the complexity is maximal in such systems.
\end{abstract}




\section{Introduction}

The definition of complexity has recently seen major improvements. It has been acknowledged for a long time that simply accounting for information (Shannon or Fisher information, for instance) does not fully grasp the notion of complexity, since a perfect chaos maximizes information but it is actually not much more complex than perfect order. As Crutchfield noted in 1994, Physics does have the tools for detecting and measuring complete order equilibria and fixed point or periodic behavior and ideal randomness via temperature and thermodynamic entropy or, in dynamical contexts, via the Shannon entropy rate and Kolmogorov complexity. What is still needed, though, is a definition of structure and way to detect and to measure it. [4].

Seth Lloyd counted as many as 40 ways to define complexity, none of them being completely satisfactory. A major breakthrough came from the definition proposed by Lopez-Ruiz, Mancini and Calbet (LMC) [9]. Although not without problems [5, 7], LMC's complexity clearly separated and quantified the contributions of entropy and structure. LMC measured structure thorough disequilibrium. Building on this proposal, Kowalski et al. [7] refined the definition of disequilibrium.

One should note that while entropy is a general concept that can be applied across a wide range of model families, this is not the case with measures of structure. With structure one needs to know what to seek. Most efforts to define disequilibrium focus on time series.

We are interested, though, in a statistical complexity measure (SCM) for models with spatial dimensions. This includes dynamical PDE-based models, such as Navier-Stokes, and Agent-Based Models (ABM), such as Collective Motion [10]. Here we use the vocable dynamical because the structures we are interested in are easily recognized (at least visually) by studying velocity fields. Other models of interest are static (i.e., not characterized by its velocity field, but rather from scalar quantities as density or spin). Examples of these models include PDE-based models such as Reaction-Diffusion, and Cellular Automata (e.g. Ising models).

Our hypothesis is that, for these systems, a good candidate for capturing the structural component in the definition of complexity is a correlation (of the velocity field in the dynamical cases, and of density or other scalar fields in the static ones). That is

$$
C(s(\hat{x}, t))=H(s(\hat{x}, t)) D(s(\hat{x}, t)),
$$

where $H$ stands for entropy (Shannon's, Fisher's, or Kullback-Leibler's), and $D$ is a correlation. $C(s(\hat{x}, t))$ represents the local statistical complexity measure, and $s(\hat{x}, t)$ is the state of the system at time $t$ in position $\hat{x}$, characterised by some scalar or vector field.

A global SCM is recovered by integration over all the simulation domain 
$\Omega:$

$$
C(s(t))=\int_{\Omega} d \hat{x} C(s(\hat{x}, t)) .
$$

$C(s(t))$ is an extensive property. An intensive property is derived by defining

$$
C_{I}(s(t))=\frac{C(s(t))}{\int_{\Omega} d \hat{x}},
$$

which is simply the average complexity field.

It is also important to acknowledge that the perception of complexity is deeply imbricated with the scale of measurement. Therefore, we should aim at measuring $C$ at different scales. The idea of studying complexity as a function of scale is not new, as represented for instance in the concepts of complexity profile [1, 2] and d-diameter complexity [3]. In comparison with these generic frameworks, we aim at exploring a definition that disentangles the contributions from entropy and structure and directly exploits the characteristics of the models under study (velocity fields and density fields in particular) as a proxy for structure.

Density fields (specifically chemical concentrations) as a proxy for structural information were studied in the case of reaction-diffusion models [8]. This work analysed the behaviour of structural information as both a spatial field and across scales. We expect this approach to be also fruitful when structure is characterised by velocity fields rather than density fields, and further enlightened by separating the contributions of noise and structure.

We aim to study the validity of this hypothesis by measuring $C(s(t))$ in numerical simulations of different models. In this context we define i) the microscale, as the size of the simulation mesh (small enough to represent the microscopical regime characterized by chaotic dynamics), ii) the mesoscale, as the scale at which a maximum of complexity is observed (typically characterised by the formation of turbulence, vortices, clusters, bands, flocks, etc.), and iii) the macroscale, large enough to reach the hydrodynamical (or equivalent) limit, typically characterized by different phases and phase transitions (ordered or chaotic, but not complex).

Representing these three scales in a single simulation is notoriously difficult in some cases (for instance, a Navier-Stokes scenario). The microscale and macroscale are separated by many orders of magnitude. In this case, the strategy should include different simulations for the distinct scales, adapting the pertinent equations to each scale and dynamics.

We find a simpler prospect in Collective Motion. Here the dynamics are characterized by three scales within a few orders of magnitude, and it is then amenable to a single simulation encompassing them all. In this preliminary study we will focus attention then in Collective Motion. 


\section{Statistical complexity measures}

Initial value problems with spatial dimensions belong fundamentally to two big families: mesh-based and meshless. The former include lattice discretizations of continuous PDE-based problems, such as Finite Volume Methods or Finite Difference Methods. It includes, as well, problems directly defined on a lattice, such as Cellular Automata (Ising models for instance). Meshless models include discretizations of PDE-based problems that use particle discretizations (such as Smoothed Particle Hydrodynamics), as well as problems directly defined on agents or particles, such as Collective Motion.

In order to define a SCM for such Initial Value Problems, we will rely on meshes of different cell sizes, defined on the problem's domain $\Omega$. In the case of mesh-based models, the actual lattice defining the simulation problem will correspond to the finest SCM mesh, while coarser SCM meshes for larger scales are trivially obtained by coarse-graining the finest SCM mesh at various scales.

In the case of meshless models, we define the cell size of the finest SCM mesh as the typical separation of two agents. The value of a certain field in a cell of this mesh is obtained by averaging the corresponding fields of the agents falling into such cell. Coarser meshes are also obtained by averaging over larger cells.

Let us see how to define a SCM, in this framework, for the case of an Agent-Based Model characterized by a velocity field (this is the family of models to which Collective Motion belongs to). That is, we consider $s=\{\hat{v}\}$. The same procedure is feasible with other fields (density, spin, and so on). One needs to properly characterize the fields that, in each case, better define the structures representing complexity.

Thus, we consider an agent in an ABM to be characterized, as a minimum, by its position and velocity. In order to study complexity at different scales we need to define meshes of various sizes. As previously stated, each cell in these meshes is characterized by the same fields as those of the ABM model, with values computed by averaging the value of the corresponding fields for all agents falling into the cell. For instance,

$$
\hat{v}_{i}=1 / M \sum_{m} \hat{v}_{m},
$$

where $\hat{v}_{i}$ is the velocity in cell $i, M$ is the number of agents in the cell, and $m$ runs over all such agents. If $M=0$, then $\hat{v}_{i}=0$.

Following [10], and re-scaling so we get a positive value, we define the velocity correlation as

$$
D^{l}\left(\hat{v}_{i}\right)=\frac{1}{N} \sum_{j \sim i} \frac{\hat{v}_{i} \hat{v}_{j}}{\hat{v}_{i}^{2}+\hat{v}_{j}^{2}}+\frac{1}{2},
$$


where $\hat{v}$ is the velocity vector and the product is scalar. $i$ defines a particular cell at scale $l$, and $j$ indexes its $N$ immediate neighbours ( 8 in 2D). For practical purposes we can only study the cases $\delta x \leq l \leq L / 4$, where $\delta x$ is the finest mesh size and $L$ is the size of the simulation domain.

This provides us with a local measure of correlation, and through (1) a local measure of complexity (a complexity field). Afterwards, we can integrate this field over all the spatial mesh in order to obtain the global complexity measure $C^{l}(\hat{v}(t))$.

In this environment we also compute $H^{l}\left(\hat{v}_{i}\right)$ as a local entropy field. We adopt the strategy defined by $\mathrm{Xu}$ et al [11]. A first step is to determine a procedure to obtain a Probability Density Function (PDF). To do so we define a partitioning of the cell velocity vectors depending on their polar angle $\theta, 0 \leq \theta \leq 2 \pi$, into a number of bins $x_{k}, k=1 \ldots n$ (for $2 \mathrm{D}$ systems we set $n=8$ ). We continue by estimating the probability for the vectors in the neighbourhood of cell $i$ as falling into each bin:

$$
p_{i}^{l}\left(x_{k}\right)=\frac{N\left(\theta_{j \sim i} \in x_{k}\right)}{N} .
$$

In these circumstances, the Shannon entropy becomes

$$
H^{l}\left(\hat{v}_{i}\right)=\sum_{k} p_{i}^{l}\left(x_{k}\right) \log _{2} p_{i}^{l}\left(x_{k}\right) .
$$

Once we have defined a local correlation field and a local entropy field, we can obtain $C^{l}(\hat{v}(t))$ as a field for any spatial position, at any time, and for any (available) scale.

\section{Objectives}

We already discussed in the introduction that Collective Motion is easier to study than more complex models such as Navier-Stokes. A first step may be to study the shape of $C^{l}$ for collective motion, that is, how complexity varies across scales, and thus confirm the hypothesis that the curve will look like the one represented in Figure 1.

The hypothesis is based on the visual insight one acquires when looking at figures 2 and 3 . These snapshots are taken from an agent simulation of a Collective Motion model. Zooming into the velocity field (Figure 3) we see how the chaotic component rules the dynamics. However, at a larger scale (Figure 22) the dynamics is characterized by clustering at a mesoscale, while isotropy emerges at larger scales (if we adequately zoom out). 


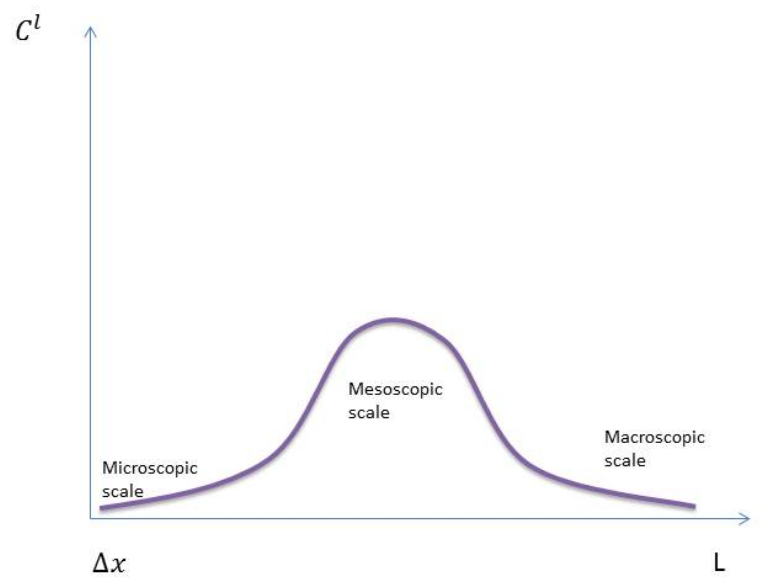

Figure 1: Expected complexity versus scale for a model exhibiting chaotic dynamics at microscale, emergence of structure at mesoscale, and tendency to isotropy at macroscale. 


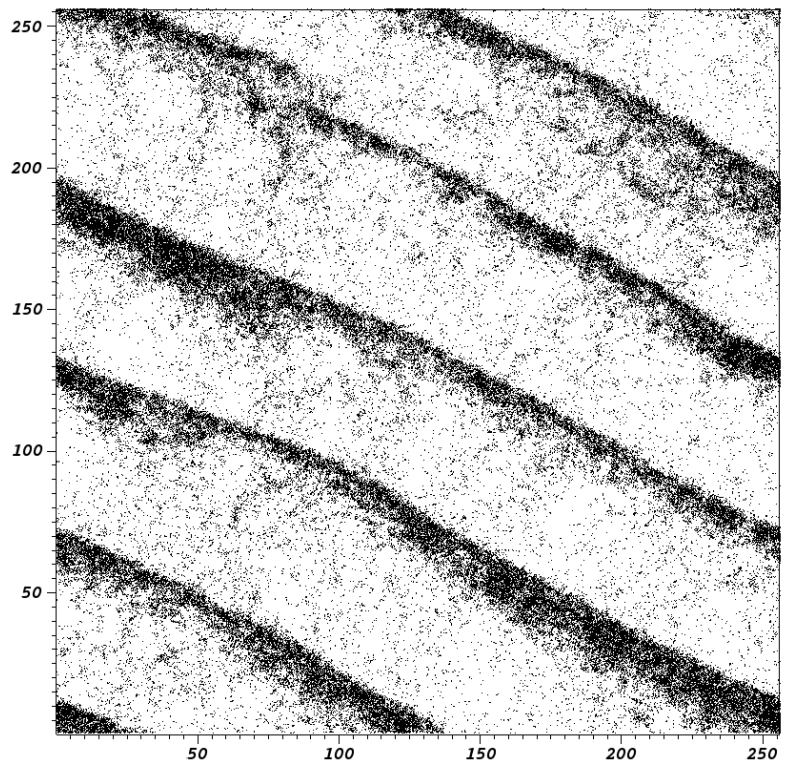

Figure 2: Agent distribution in space for a 131.072 particle simulation of a collective motion model. The model implemented is the vectorial noise model proposed by Gregoire and Chaté [6], with domain size $L=256$, density $\rho=2$, noise level $\eta=0.611$, speed $v_{0}=0.5$, and time step $\Delta t=1$. In this snapshot the system has evolved to a stationary state characterized by high-density bands travelling in a certain direction. 

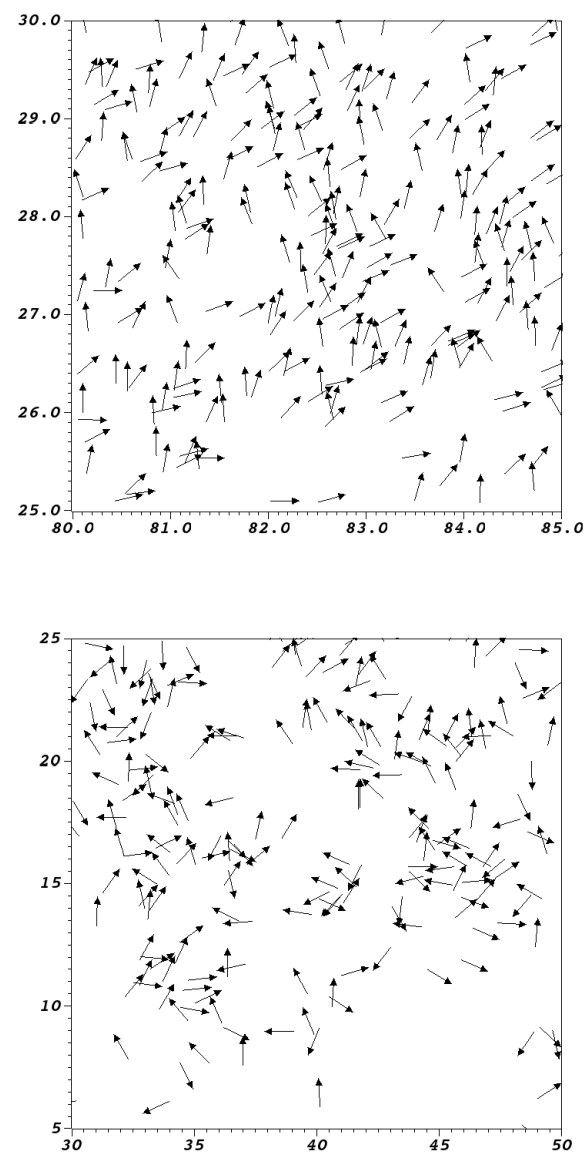

Figure 3: Zoom on two small regions of the same simulation represented in Figure 2. Arrows correspond to the velocity of each agent. The top figure zooms into a band; notice the preferred directions North and Northeast. The bottom figure zooms into an inter-band space, and shows a chaotic pattern of velocities. 


\section{Results}

We have computed the normalised entropy, velocity correlation and complexity fields for the snapshot considered in Figure 2. In Figure 4 we show the results for the finer scale ( $\Delta x$, taken as the typical inter-agent distance). Notice the complementary pattern of velocity correlation and Shannon entropy. The former is maximal inside the bands, corresponding with a relatively homogeneous velocity field. The latter is maximal within the inter-band regions, particularly in zones when the velocity field is more disordered. By multiplying these fields, the complexity fields highlights the areas with a balance between chaos and order, which correspond with certain locations of the bands (typically on the band edges, where they make a transition to the turbulent inter-band regions). Therefore, in general neither the innermost parts of the bands (highly ordered) nor the inter-band regions (highly disordered) show the maximum complexity, but rather the areas that represent a transition between them.

We have also analysed how complexities changes with scale. To discard effects produced by the domain size we have run 4 simulations of sizes $256 x 256,512 x 512,1024 x 1024$, and $2048 x 2048$. We have taken snapshots every 500 time steps from $t=20.000$ (where the system is already in a stationary) to $t=30.000$. For each snapshot and each scale we have computed the complexity field. We represent in Figure 5 the average complexity (averaging both over space within a snapshot and over the set of snapshots) versus scale. From the comparison among the four plots we see that the domain size is not affecting the structure of the stationary state.

The complexity is maximum in the range $3 \Delta x-20 \Delta x$, approximately. The typical band width is about $20 \Delta x$, while the transition zone between bands and inter-band regions has a width of a few $\Delta x$. Thus, the limits of the mesoscopic scale in this system and the range where complexity as defined in (3) is maximal are barely the same.

\section{Conclusions}

Neither measures of entropy nor measures of structure, taken separately, account for the accepted notion of complexity. Following the now quite established trend of combining both quantities into definitions of statistical complexity measures, we have explored a definition of complexity for spatial dynamical systems. Our hypothesis is that Shannon entropy is a good proxy for the component of complexity contributed by disorder, while correlation (velocity correlation in particular) accounts well for structure.

We have tested our ansatz against the vectorial noise model of Collective Motion. This is a system with a mesoscale characterised, in the stationary 

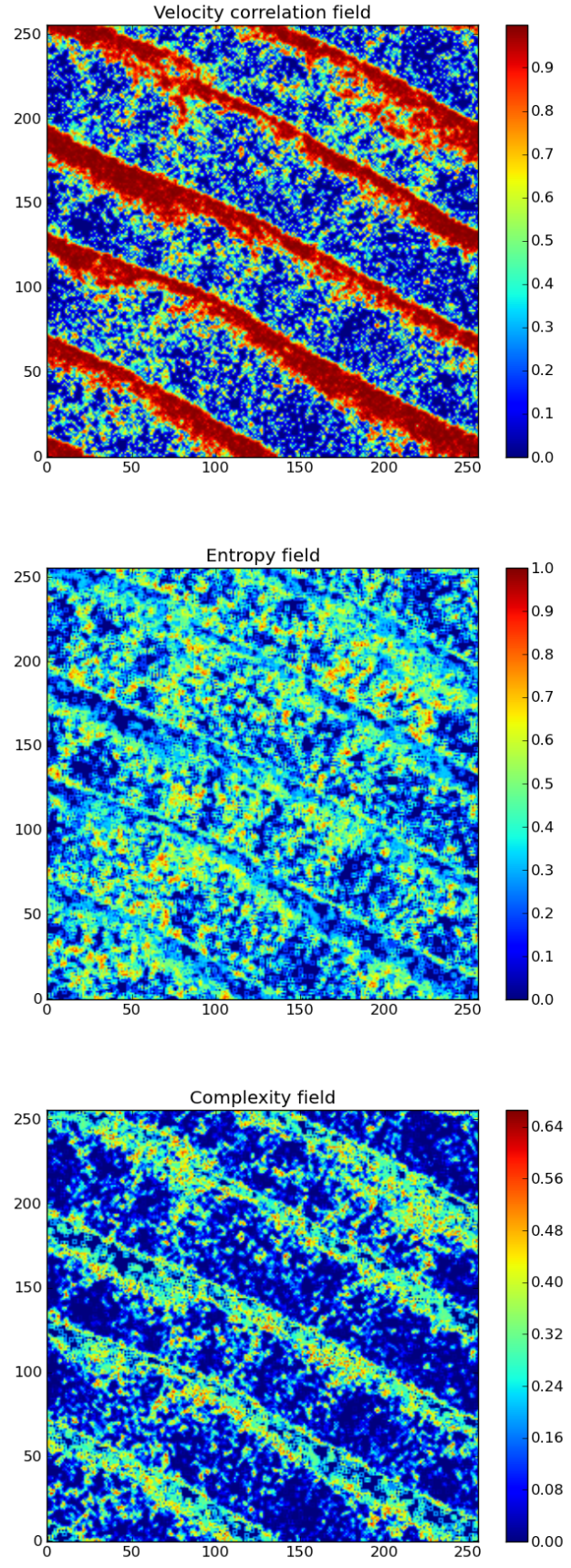

Figure 4: Velocity correlation, entropy, and complexity fields for the snapshot corresponding to Figure 2. 


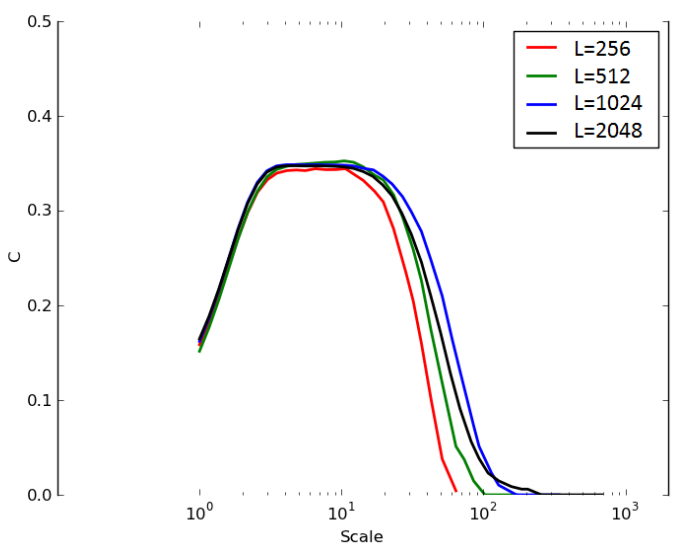

Figure 5: The average complexity versus scale of a stationary solution for the Gregoire and Chaté vectorial noise model. The different lines correspond to 4 different domain sizes: $L=256, L=512, L=1024$, and $L=2048$.

limit, by travelling ordered bands separated by chaotic inter-band regions. We have shown that the proposed definition of complexity field is low both in the most ordered regions, this is the inner part of the bands, and the most disordered regions (the inter-band regions). The complexity field is maximum in the band edges, when the ordered dynamics of the bands meets the chaotic environment of the inter-band regions. This is precisely the behaviour we expect in a SCM.

We have also studied the average complexity which characterises the different scales in this system, and found that the complexity is minimum at both the microscale and the macroscale, a property we were expecting from a reasonable definition of complexity. For the typical scales that characterize the mesoscale, we find that the complexity is maximal, in accordance with our hypothesis.

With these considerations, we conclude that the proposed definition of statistical complexity measure for a spatial dynamical system is quite reasonable, and it is a candidate measure for detecting the mesoscale of arbitrary dynamical systems as well as regions where complexity is maximum. As a follow-up, we will consider the behaviour of the proposed definition of complexity for additional spatial dynamical systems. We will also extend our study to systems characterised by other variables other than velocity, such as spin or density. 


\section{Acknowledgements}

We thank Miquel Trias (Google) for initial feedback on the definition of a SCM for spatial fields. We thank Joan Massó (University of the Balearic Islands) for useful discussion on the domain size analysis. We thank Rick Quax (University of Amsterdam) for illuminating references that improve the contextualization of our work. The research leading to these results has received funding from the European Union Seventh Framework Programme (FP7/2007-2013) under grant agreement no 317534 (the Sophocles project).

\section{References}

[1] Yaneer Bar-Yam. Complexity rising: From human beings to human civilization, a complexity profile. Encyclopedia of Life Support Systems (EOLSS), UNESCO, EOLSS Publishers, Oxford, UK, 2002.

[2] Yaneer Bar-Yam. Multiscale complexity/entropy. Advances in Complex Systems, 7(01):47-63, 2004.

[3] Gregory J Chaitin. Toward a mathematical definition of life. In The Maximum Entropy Formalism, R.D.Levine and M.Tribus (eds). MIT Press, 1979.

[4] James P. Crutchfield. The calculi of emergence: computation, dynamics and induction. Physica D: Nonlinear Phenomena, 75(13):11 - 54, 1994.

[5] David P Feldman and James P Crutchfield. Measures of statistical complexity: Why? Physics Letters A, 238(45):244 - 252, 1998.

[6] Guillaume Grégoire and Hugues Chaté. Onset of collective and cohesive motion. Phys. Rev. Lett., 92:025702, Jan 2004.

[7] Andres M. Kowalski, Maria Teresa Martin, Angelo Plastino, Osvaldo A. Rosso, and Montserrat Casas. Distances in probability space and the statistical complexity setup. Entropy, 13(6):1055-1075, 2011.

[8] Kristian Lindgren, Anders Eriksson, and Karl-Erik Eriksson. Flows of information in spatially extended chemical dynamics. In Proceedings of ALife, volume 9, 2004.

[9] R. Lopez-Ruiz, H.L. Mancini, and X. Calbet. A statistical measure of complexity. Physics Letters A, 209(56):321 - 326, 1995.

[10] Tamas Vicsek and Anna Zafeiris. Collective motion. Physics Reports, 517(34):71 - 140, 2012. 
[11] Lijie Xu, Teng-Yok Lee, and Han-Wei Shen. An information-theoretic framework for flow visualization. Visualization and Computer Graphics, IEEE Transactions on, 16(6):1216-1224, 2010. 